\title{
Universo invisible: una aproximación al conocimiento de la cultura Jama Coaque a través del análisis de dos vasijas cerámicas del Museo de América
}

\author{
Andrés GutiÉrRez UsiLlos \\ Museo de América, Madrid \\ andres.gutierrez@mecd.es
}

Recibido: 13 de septiembre de 2013

Aceptado: 30 de septiembre de 2013

\section{RESUMEN}

Los análisis arqueométricos permiten descubrir rasgos y trazas no sólo sobre componentes, estructura o fabricación de los objetos arqueológicos, sino que también posibilitan la interpretación sobre el modo de vida de las culturas antiguas. Radiografías, sondas con videocámara, junto con el análisis de los restos orgánicos que contienen o las interpretaciones iconográficas sugieren datos de gran interés para conocer un poco más en profundidad la cultura Jama Coaque del Ecuador prehispánico.

Palabras clave: Jama Coaque, Museo de América, cerámica, arqueometría, radiografía, botella silbadora, SEM-EDX, cromatografía de gases-espectrometría de masas, FTIR, almidones, pólenes.

\section{Invisible Universe: An Approach to Jama Coaque Culture Through the Analysis of Two Ceramic Vessels at the Museo de América}

\begin{abstract}
Archaeometric analyses allow us to discover traces and features not only about components, or manufacturing structure of archaeological objects; they also make possible the interpretation of lifestyles of ancient cultures. Radiographs, probes with camcorder, along with the analysis of organic remains contained or iconographic interpretations suggest very interesting data to know more in depth the Jama Coaque culture of pre-Hispanic Ecuador.
\end{abstract}

Key words: Jama Coaque, Museo de América, archaeometry, X-ray, whistling bottle, SEM-EDX, Gas chromatography-mass spectrometry, FTIR, starch, pollen.

Sumario: 1. Introducción. 2. «Descubriendo» el interior de las vasijas Jama Coaque. 3. Datación. 4. Los pigmentos y componentes. 5. Análisis e identificación del contenido de la botella. 6. La muestra del interior. 7 . Interpretación. 8. Referencias bibliográficas.

\section{Introducción}

Atrás quedaron las reflexiones de Ian Hodder y la respuesta de Alcina Franch sobre el valor de los materiales arqueológicos conservados en los museos y la falta de contextos (Gutiérrez Usillos 2010: 68-69). Es obvio que, aunque en la mayor parte de los casos no puedan conocerse de forma directa cuáles fueron las circunstancias en que estos objetos se depositaron o se recuperaron del yacimiento, la propia información que contienen es sumamente valiosa para la comprensión de las culturas que la produjeron. Además, es posible una «recontextualización» de esos objetos, a partir de otros similares, de documentos o de otro tipo de información contextualizada. Y no se trata en este caso únicamente de comparaciones estilísticas o iconográficas, cuyo 
valor ha sido ya resaltado (Gutiérrez Usillos 2011), sino de rescatar información arqueológica o arqueométrica, similar a la que se recupera a partir de las excavaciones, con la limitación de carecer de estos contextos primarios.

Este universo invisible en el ámbito de las cerámicas también engloba los materiales conservados en museos, por lo que deberían poder aplicarse los mismos tipos de análisis. Y para ello, se han de aplicar las mismas técnicas arqueométricas, metodologías de estudio generalmente desarrolladas por ciencias auxiliares de la propia arqueología, ya sea a partir de análisis físicos o químicos, que posibilitan el estudio del pasado. El fin último de estos análisis es saber más, no sólo sobre la dieta o la técnica de fabricación, sino sobre la vida cotidiana de las culturas del pasado y conocer cómo se resolvían los mismos problemas que hoy día afrontamos: producción, transformación, intercambio, conservación de alimentos o bebidas, usos sociales y división de género/edad, usos rituales y simbólicos, o tipos de productos utilizados, entre otros.

Por lo tanto, las técnicas para este conocimiento de objetos de museos no son nuevas. Son las mismas que se vienen aplicando en investigaciones sobre materiales recuperados de excavaciones arqueológicas contextualizadas y que pretenden descubrir e interpretar información que va más allá de la estrictamente tipológica, cronológica o clasificatoria. Por ejemplo, cerámicas que se han utilizado para la cocción de alimentos o para contener productos como aceites o grasas, conservan en la porosidad de sus paredes interiores partículas orgánicas, granos de almidón, fitolitos o levaduras, que posibilitan la interpretación de esa utilización concreta de las mismas. Investigaciones que incluso han conseguido recuperar o «volver a la vida» levaduras de fermentación de los azúcares para convertirlos en alcohol, entrando ya en lo que se viene llamando la arqueología microbiana (ver Carvajal y Portero 2012).

Con este artículo, nuestro objetivo es poner de manifiesto el tipo de información que es posible recuperar a partir del análisis realizado sobre dos vasijas cerámicas descontextualizadas, pertenecientes a la Cultura Jama Coaque y conservadas en el Museo de América en Madrid (MAM 1991/11/16 y MAM 3736) como aporte de información substancial en torno a aspectos ceremoniales de esta cultura, que en algún caso incluso, no han sido hallados en otros contextos arqueológicos documentados. El objetivo de estos estudios es ir recomponiendo información suficiente para reconstruir y entrelazar el disperso mosaico del conocimiento sobre esta cultura.

Jama Coaque es una de las culturas prehispánicas desarrolladas en el actual territorio del Ecuador, que destaca por la complejidad y estética de sus cerámicas. Esto explica su alta presencia en colecciones y museos de todo el mundo, hasta el punto de encontrarse en la lista roja de bienes culturales en peligro elaborada por ICOM ${ }^{1}$. A pesar de su difusión, es realmente muy poco lo que conocemos de esta cultura (Gutiérrez Usillos 2011), cuya extensión cronológica muestra una pervivencia de rasgos extraordinaria, desde el 350 a.C. hasta 1532 d.C., pues abarca dos períodos cronológicos, conocidos como Desarrollo Regional e Integración.

1 Ver http://icom.museum/recursos/banco-de-datos-de-las-listas-rojas/categoria/figuras-y-vasijas-jama-coaque/L/1/ (con acceso el 29 de julio de 2012). 


\section{2. «Descubriendo» el interior de las vasijas Jama Coaque}

El primer paso en la aproximación al estudio de las cerámicas suele ser el análisis visual de las mismas. La forma, la técnica de fabricación y decoración o la iconografía son descripciones habituales en las fichas de documentación y catalogación de estos objetos y quedan al margen de este estudio. Sin embargo, no es frecuente prestar la misma atención al interior, muchas veces dando por supuesto que no contienen evidencias o que si las contuvieran estarían tan contaminadas que serían inútiles o carecerían de interés. Sin embargo, conocer el interior de las vasijas, especialmente las de formas cerradas como botellas, resulta esencial, pues realmente se trata de su parte funcional y la que, en caso de existir, guardará las evidencias de su uso.

Para acceder al conocimiento del interior de estas vasijas Jama Coaque hemos utilizado varias técnicas. Cada una de ellas permite conocer una parte de la información específica que se trata de localizar y que no es visible de forma inmediata: para la estructura y composición por ejemplo se cuenta con la técnica radiográfica, para comprobar qué restos contiene hemos utilizado una sonda con videocámara, y con el fin de identificar de qué se trata ese contenido, se ha realizado una toma de muestras y su posterior análisis en laboratorio.

\subsection{Universo invisible 1: el interior de la vasija a través de la radiografía}

Una de las cerámicas más significativas de la cultura Jama Coaque, del conjunto que se conserva en el Museo de América es esta representación de un guerrero (Figura 1). Se trata de una tipología que se repite con cierta frecuencia en esta cultura y que muestra invariablemente un personaje masculino, ataviado con tocado, taparrabos y en ocasiones camiseta abierta o poncho, portando un escudo en una mano y en actitud de arrojar un dardo con la estólica o una lanza corta en otros casos. Esta escultura antropomorfa está hueca, muestra profusión de aplicaciones plásticas e intensa policromía y está por lo general unida mediante un puente comunicante a un vaso cilíndrico más o menos alto. Es decir, se trata de vasijas de uso ceremonial, de elaboración muy compleja ${ }^{2}$.

El uso del molde en la producción de figuritas de esta cultura es un hecho ya contrastado (Gutiérrez Usillos 2011: 71-73). Realizada por partes, cada una de ellas se ensamblaría posteriormente para formar la figura completa. En la mayoría de los casos, las aplicaciones posteriores, el alisado y la cocción final no permiten apreciar cómo se producía esa unión y esto se hace evidente a partir de la imagen radiográfica ${ }^{3}$ (Figura 2). La cabeza, realizada a molde y completada con aplicaciones decorativas -orejeras, nariguera y tocado-, habría sido fabricada por separado y luego insertada en el cuerpo mediante un vástago que es el propio cuello de la cabeza y que se prolonga en el interior de la vasija varios centímetros (Figura 2, sector 3). Los brazos

\footnotetext{
2 En otra ocasión hemos hecho una sugerencia de interpretación y una contextualización de este tipo de representaciones (Gutiérrez Usillos 2011: 45-50).

${ }^{3}$ La radiografía ha sido realizada por el equipo técnico del Instituto de Patrimonio Histórico Español. Agradecemos a Miriam Bueso y a Tomás Antelo del IPCE por su colaboración. Y muy especialmente a Gonzalo Trancho por haber realizado los ajustes a la imagen para poder visibilizar diversos detalles.
} 

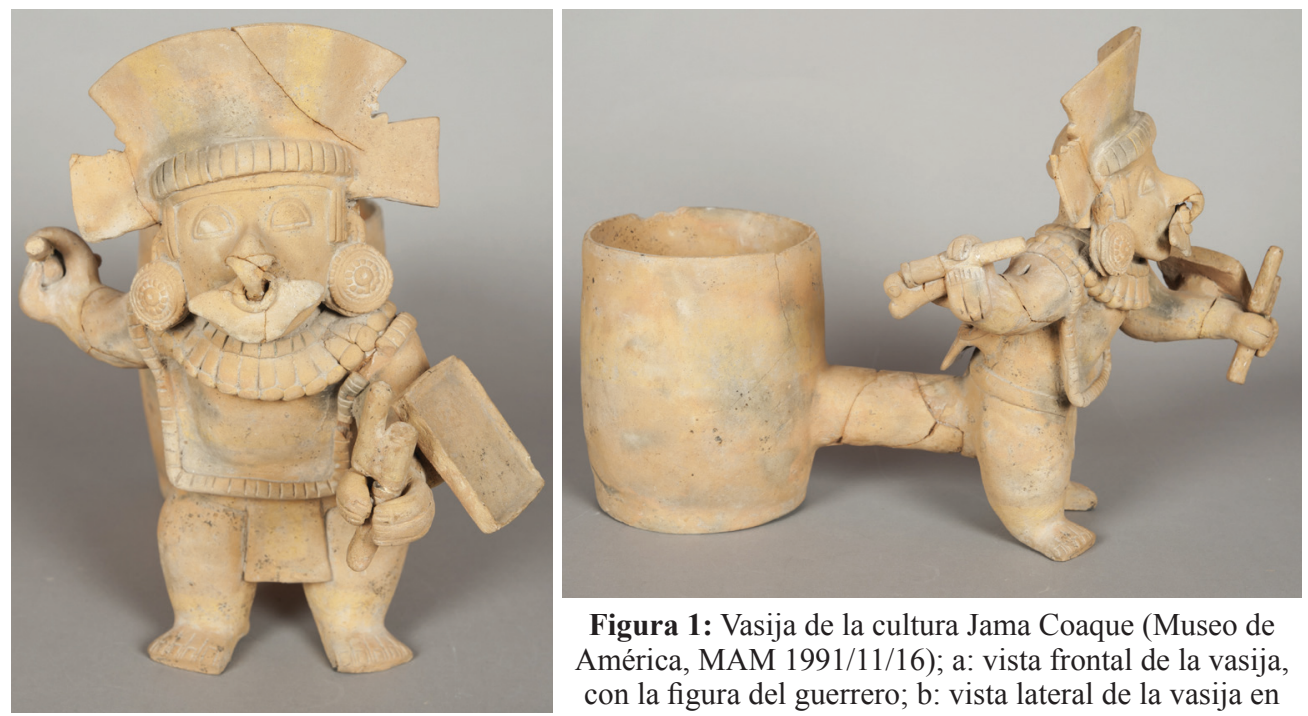

Figura 1: Vasija de la cultura Jama Coaque (Museo de América, MAM 1991/11/16); a: vista frontal de la vasija, con la figura del guerrero; b: vista lateral de la vasija en donde se aprecia la hendidura en el brazo.

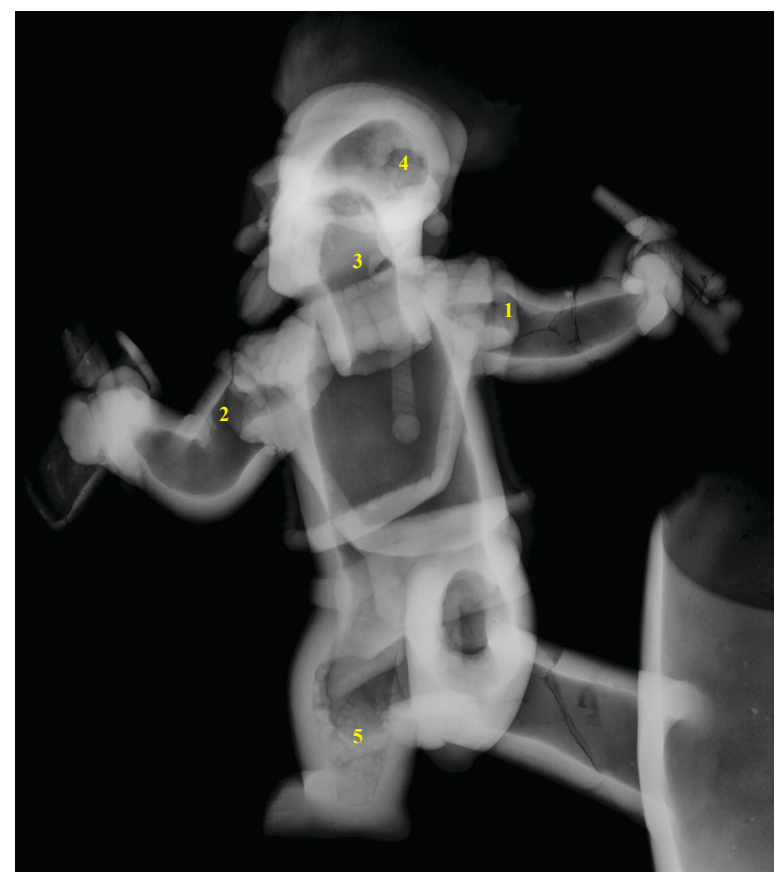

Figura 2: Radiografía de la vasija MAM 1991/11/16. «1» y «2»: aplicaciones para adherencia de los brazos; «3»: cuello encajado en el cuerpo; «4»: cabeza rellena de tierra; $« 5 »$ : piernas rellenas de tierra.

han sido construidos y aplicados también aparte. En este caso, parece que para dotar a la pieza de mayor estabilidad, a la altura de los hombros, el cuerpo presenta unas prolongaciones cilíndricas -apreciables en forma en $U$ con el lado abierto hacia los extremos (Figura 2, sector 2)-, que servirían como apoyo o base para la fijación de los brazos al cuerpo. 
La figura antropomorfa de esta vasija presenta un par de orificios rectangulares en los brazos y otro de mayor tamaño bajo la nuca, en la parte posterior de la cabeza. Este último nos indujo a pensar que se trataba de una vasija silbadora y que, por tanto, al verter el líquido en el vaso cilíndrico, el aire se desplazaría por el puente hasta la figura, provocando la salida del mismo a través de los orificios ${ }^{4} \mathrm{y}$ emitiendo un sonido que sería característico de esta tipología de vasijas. Desconocemos si los orificios sobre los brazos servirían también para provocar nuevos tonos, al obstruirlos o liberarlos con los dedos de las manos al tiempo que se movía la vasija. En todo caso, no es el único ejemplar que muestra estas ranuras en los antebrazos ${ }^{5}$ que no parece una ranura de cocción por encontrarse en lugar visible y por no ser algo generalizado en todas estas vasijas. La interpretación del uso de las ranuras como sistema de modular el sonido permite entender la forma de manipulación ceremonial de esta vasija, apoyando el vaso entre el brazo y el cuerpo del que la maneja y sosteniendo la figura por los brazos de la misma, de forma que con sólo dos dedos, posiblemente el pulgar de cada mano, puedan taparse o abrirse los mencionados orificios, mientras se hace oscilar la vasija para mover el líquido y que el aire penetre en la cámara, produciéndose el silbido por el cambio de presión.

En la radiografía no se distingue la existencia de cajas de resonancia (sordinas) donde se produce la reserva de aire. ¿Todo el cuerpo del guerrero funciona como cámara de resonancia? El canal de unión entre el vaso y la figura es un conducto cuya longitud permite «prolongar el sonido y obtener interesantes sonidos de «respiración» intercalados» (Pérez de Arce 2004: 15). Lo más común en estas figuras es que la propia cabeza o cuerpo funcionen como cámara de aire.

Esta cerámica Jama Coaque, por lo tanto, es una vasija silbadora similar a las que se encuentran desde México hasta Perú. En Ecuador se reseñan ejemplos en diversas culturas, desde el Formativo, generalmente con un silbato en el gollete o en el cuello que se activa soplando por el mismo. Estas vasijas silbadoras de culturas Machalilla o Chorrera (Formativo Medio y Final, entre1500 y 500 a.C.) a diferencia de ésta, cuentan con la presencia de un pequeño silbato o sordina, una cámara de resonancia similar a las que se aprecian en las radiografías de las figuras Bahía estudiadas por Mónica Gudemos (2009).

Sin embargo, Jama Coaque no continúa esa tipología de vasijas silbato sino que se decanta por otra forma en la que el sonido se emite por el desplazamiento del aire al mover el líquido de su interior. Otra vasija silbadora Jama Coaque, similar a ésta del Museo de América, se ha documentado en el Museo Chileno de Arte Precolombino (MCHAP 0050). Dicha vasija presenta también el orificio en la parte posterior de la cabeza (de $0,9 \times 0,4 \mathrm{~cm}$ ), donde se aloja el silbato sin sordina que emite un tono o sonido grave (Do). Esta vasija, al igual que otra del mismo tipo (MCHAP 0049) en este caso representando a un guerrero, emite como la anterior un sonido «grave y pausado». Cada botella produciría un tono, melodía o un sonido diferente, buscándose una relación entre el objeto, lo que este representa y el ritual para el que se utilizaría.

\footnotetext{
4 Dimensiones de los orificios: nuca, $2 \mathrm{~cm}$ de largo x 0,6 alto; brazo izquierdo y derecho, $1 \mathrm{~cm}$ de largo x 0,2 de ancho.

5 En la exposición permanente del Museo del Centro Cultural Libertador Simón Bolívar, en Guayaquil (GA 1/1157/79) al menos, hemos visto otro ejemplar con estos mismos orificios en los brazos.
} 


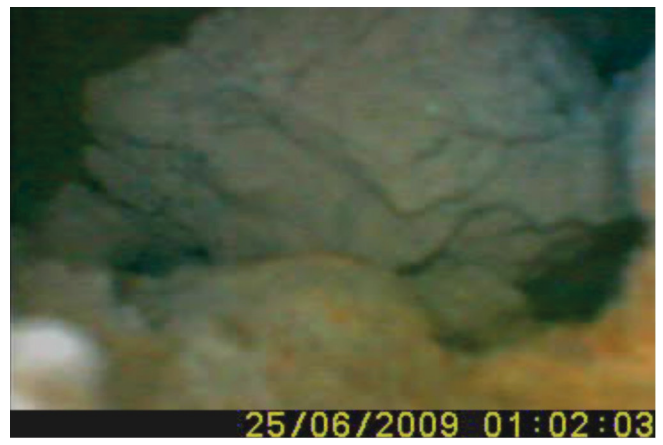

Figura 3: Vista del interior de la cabeza del guerrero de la vasija MAM 1991/11/16, tras haber provocado el desprendimiento de una pequeña muestra de tierra.

Aunque lo frecuente de las vasijas silbadoras es un sonido agudo o alto, en estos casos, las vasijas Jama Coaque son las únicas que presentan un sonido grave, producido en las grandes «ocarinas» que conforma la propia cabeza de la figura. El efecto sico-acústico de estas botellas silbadoras era un complemento fundamental en las ceremonias en las que se consumían plantas psicoactivas, como técnicas chamánicas de éxtasis (Pérez de Arce 2004).

Además de la estructura, en la radiografía de la vasija del Museo de América (Figura 2, sectores 4 y 5) también se distingue la presencia de un relleno tanto en la cabeza como, sobre todo, en la parte inferior de las piernas de la figura. En la radiografía no se distingue de qué se trata, así que procedimos a la segunda toma de datos, a través de la sonda de la vasija, a fin de conocer qué es lo que encierra el interior de la figura antropomorfa de esta vasija.

\subsection{Universo invisible 2: el reconocimiento del interior con sonda y videocámara}

Para visualizar el contenido de esta figura cerámica hemos utilizado una sonda con videocámara accediendo tanto a través del orificio de la nuca para comprobar el interior de la cabeza, como a través del conducto de unión de la vasija y la figura para llegar hasta las piernas. En este caso se trataba tan sólo de una acumulación de tierra muy fina, de color grisáceo, y aspecto ceniciento que se había depositado en la base de la figura, rellenando hasta la mitad de la pierna, y adherido al interior de la cabeza. Se tomaron muestras para posteriores análisis (Figura 3).

\subsection{Universo invisible 3: la toma de muestras y su análisis}

La analítica de objetos de la cultura Jama Coaque se complementa con la toma de muestras halladas en el interior de otra botella de la misma cultura que también forma parte de los fondos del Museo de América. Se trata de una cerámica globular (Figura 4), que corresponde a una de las tipologías frecuentes del ajuar ceremonial cerámico de la cultura Jama Coaque, de reducido tamaño, con cuello alto y recto, generalmente muestran representación fitomorfa en los costados. A diferencia de otras botellas similares, esta vasija presenta un personaje antropomorfo masculino sedente unido al cuerpo de la misma. 

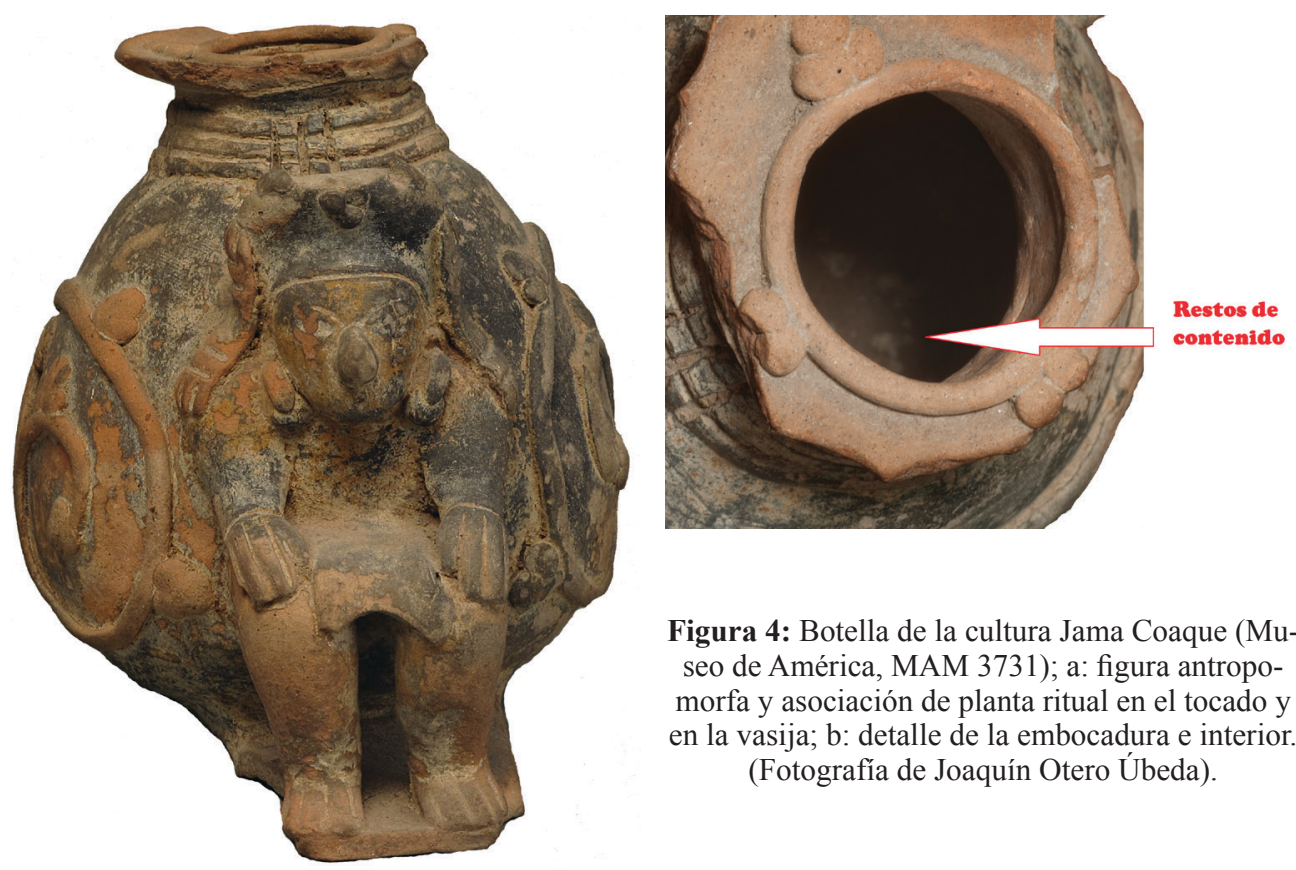

La abundancia con que se encuentran estas botellas en diversos museos hace pensar que se trataba de un objeto de uso o distribución frecuente, probablemente recuperadas como acompañamiento del ajuar funerario, aunque no se ha constatado la existencia de un contexto arqueológico asociado. Es razonable pensar también que ha de existir un vínculo entre el tipo de decoración fitomorfa, que se repite casi invariablemente en estas botellas, y el líquido que estas contuvieran.

Sin duda, por su forma se trata de recipientes para líquidos y, a juzgar por su reducido tamaño, parecen destinadas a un uso individual y ocasional. En un análisis visual inicial se observa cómo la estrecha embocadura de estas botellas presenta una doble tipología que responde a las dos formas de taponar la misma, bien con capuchón de cerámica o bien con tapón vegetal (Gutiérrez Usillos 2011) ${ }^{6}$ como en este caso. El engrosamiento del labio evertido permitiría reforzar el borde frente a la presión que se produce durante la inserción del tapón al tiempo que permitiría una mejor sujeción de la botella para la extracción del mismo.

Dado que la forma de la botella ${ }^{7}$ es ahusada, para calcular el volumen de líquido que contendría hemos tomado la fórmula de referencia para formas elipsoides ${ }^{8}$, por lo que la fórmula sería: $1,3333 * 3,141592 \times 3 \times 4,8 \times 1,2=124,6552 \mathrm{~cm}^{3}$. Por tanto, la capacidad como contenedor de la botella equivaldría a unos $125 \mathrm{ml}$ aproximadamente. Realmente es un volumen reducido para tratarse de un contenedor de bebida,

6 Tapones o «bototos» de tela con arcilla y brea probablemente para calabazos, se han documentado en la región más seca de la provincia del Guayas (Zevallos 1995: 184, lám. 55). En este caso, el tejido ha desaparecido pero conserva la impronta en el tapón de brea que además muestra el estrechamiento para ajustarse al borde.

7 La vasija tiene una altura de 9,8 cm. y un ancho máximo de 9,1 cm. El diámetro de la embocadura es de 2,4 cm.

8 Multiplicando las tres longitudes de radio de los extremos y centro de la forma elipsoidal [ $\frac{4}{3} x \pi x r 1 x r 2 x r 3$ ]. 
teniendo en cuenta, por ejemplo, que supone casi la tercera parte del contenido de una lata de refresco que suele tener $330 \mathrm{ml}$.

\section{Datación}

La fecha de fabricación de esta botella (MAM 03731) ha sido datada por termoluminiscencia $^{9}$ en una antigüedad de 651 años $( \pm 52)$, es decir, que correspondería a la segunda mitad del siglo XIV después de Cristo, en la fase final del período de Integración ${ }^{10} \mathrm{y}$ por tanto en la fase Muchique IV (1250-1580 d.C.), poco antes del fin de esta cultura.

\section{Los pigmentos y componentes}

Los análisis realizados ${ }^{11}$ en el Instituto del Patrimonio Cultural de España (IPCE) con las muestras tomadas de varias piezas Jama Coaque, entre ellas esta vasija MAM 3731, demuestran que los colores utilizados para la decoración de figuras y vasijas cerámicas (rojo, amarillo, verde y blanco) proceden de arcillas o tierras de diversos tonos y ninguno es de tipo orgánico. El informe elaborado por María Antonia García y Pedro Pablo Pérez (2011) sobre esta botella se basa en tres tipos de análisis:

- Estudio morfológico, a través de secciones estratigráficas transversales de la micromuestras en resina incolora pulida hasta obtener la sección transversal definida y analizada al microscopio óptico con luz reflejada y polarizada e iluminación UV.

- Identificación de componentes inorgánicos a través de microscopía electrónica de barrido-microanálisis por dispersión de energías de rayos X (SEM-EDX).

- Determinación de barnices y aglutinantes a través de espectrometría de infrarrojos por transformada de Fourier (FTIR).

- Identificación de aceites secantes, resinas terpénicas ${ }^{12}$ y ceras por cromatografía de gases-espectrometría de masas.

La vasija en cuestión (MAM 3731) no presenta prácticamente restos de policromía. Se han tomado muestras en las piernas, del relleno de las incisiones que marcan los dedos del pie, y alrededor de las aplicaciones de elementos vegetales y del rostro

\footnotetext{
9 Datación por termoluminiscencia de cerámicas de la cultura Jama Coaque en el Museo de América, realizada en el Laboratorio de Datación y Radioquímica, de la Facultad de Ciencias de la Universidad Autónoma de Madrid, con la colaboración del Instituto de Patrimonio Cultural de España.

${ }^{10}$ Es necesario poner en común un sistema de información conjunta entre las instituciones que custodian estas colecciones con los informes y las dataciones absolutas realizadas con estos objetos, a fin de contrastar informaciones y avanzar en el conocimiento de esta cultura o de otras del Ecuador Prehispánico.

${ }^{11}$ Hemos utilizado microscopía electrónica de barrido para identificar componentes inorgánicos, espectrómetro de infrarrojos mediante transformada de Fourier (FTIR) para determinar aparejos, barnices y aglutinantes y cromatografía de gases, utilizando un GC-MS QP5050 Shimadzu, para identificar aceites secantes, ceras y resinas terpénicas. La metodología y técnicas se encuentran detalladas en el informe realizado por María Antonia García y Pedro Pablo Pérez (2011) ( $n^{\circ}$ IPCE 30661).

12 Los terpénicos son lípidos compuestos de aceites esenciales presentes en productos destilados de flores o frutos.
} 

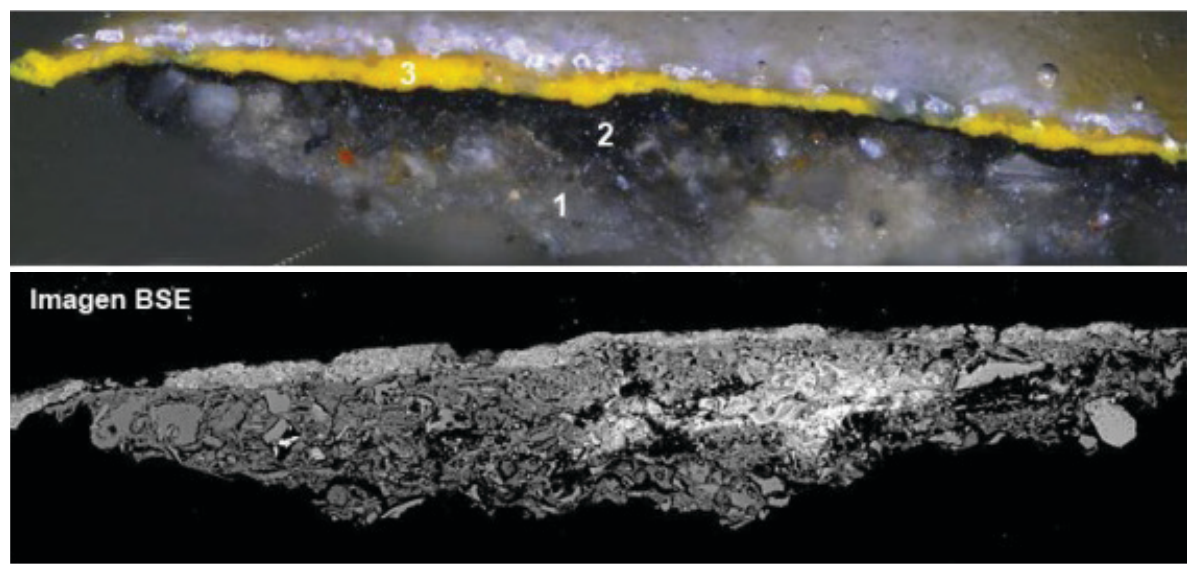

Figura 5: a: Capa amarillenta-anaranjada de la vasija MAM 3731 (García y Pérez 2011); b: imagen BSE SEM EDX de la misma vasija (García y Pérez 2011).

entre el ojo y la oreja, donde se conservan algunos restos de pintura. Estas muestras, una vez preparadas, se analizan con microscopio óptico ${ }^{13}$. Y, mediante la técnica de microscopía electrónica de barrido-microanálisis por dispersión de energías de rayos $\mathrm{X}(\mathrm{SEM}-\mathrm{EDX})$ se identifican los componentes, determinándose que se trata de tierras blancas, arcillas y ocre, de origen mineral.

El soporte cerámico es una arcilla ferruginosa con pequeñas cantidades de carbonato de calcio y fosfato cálcico, así como silicio $(\mathrm{Si})$, oxígeno $(\mathrm{O})$, aluminio $(\mathrm{Al})$, carbono $(\mathrm{C})$, potasio $(\mathrm{K})$, hierro $(\mathrm{Fe})$, calcio $(\mathrm{Ca})$ y en menor cantidad, cloro $(\mathrm{Cl})$, titanio (Ti) magnesio (Mg), sodio (N) y fósforo (P) (García y Pérez 2011: 6). Parece que los pigmentos se aplicaron directamente sobre la superficie cerámica, sin un engobe previo, aunque esto también puede deberse al desgaste de la superficie y las muestras tomadas.

El pigmento de mayor interés es el del rostro, de color amarillo-anaranjado, que, como otras vasijas similares, posiblemente sugiriera una pintura facial del individuo, ya que el amarillo parece un color utilizado en ciertas ceremonias (Gutiérrez Usillos 2011: 232 y ss.).

En la estratigrafía (Figura 5a) se observan 3 capas diferentes. La capa amarilla anaranjada (3), en la parte superior, es una policromía realizada con tierra amarilla (ocre), óxidos de hierro, tierras y fosfato cálcico en baja proporción. La capa intermedia es de color negruzco (2), formada por tierras, óxidos de hierro, carbonato de calcio y fosfato cálcico en baja proporción. Y la capa inferior (1) corresponde ya al soporte de arcilla ferruginosa, carbonato de calcio y fosfato cálcico en baja proporción.

En el análisis elemental por SEM-EDX de la misma muestra no se evidencia una diferencia morfológica apreciable ni tampoco en la composición, entre los estratos 1 y 2 (Figura 5b). La diferencia de coloración entre estas dos capas, con el oscurecimiento de la segunda podría deberse a un exceso de temperatura en el que se ha

13 Olimpus BX51, provisto de luz reflejada y polarizada e iluminación UV. 
quemado la parte más externa del soporte cerámico, antes de la aplicación de la policromía.

\section{Análisis e identificación del contenido de la botella}

La botella globular presenta también una figura antropomorfa sentada, adherida por la espalda y por la base a través de una tira o plataforma cerámica que le confiere mayor estabilidad, ya que solo permite caer la vasija hacia los lados. Sin duda, al haberse caído sobre el lateral derecho, el líquido que contuviera se fue decantando sobre el fondo cóncavo de dicho lateral depositando contra la pared unas concreciones blanquecinas.

Una vez extraída la muestra (Figura 6), se procedió a su análisis, contando inicialmente con los especialistas del Instituto de Patrimonio Cultural de España (IPCE). Para ello, se colocó la muestra en un stub de microscopía, recubriéndolo con una delgada película de oro, a fin de realizar un primer análisis con el microscopio electrónico. En las imágenes se distingue la estructura que conforma la muestra, con ca-

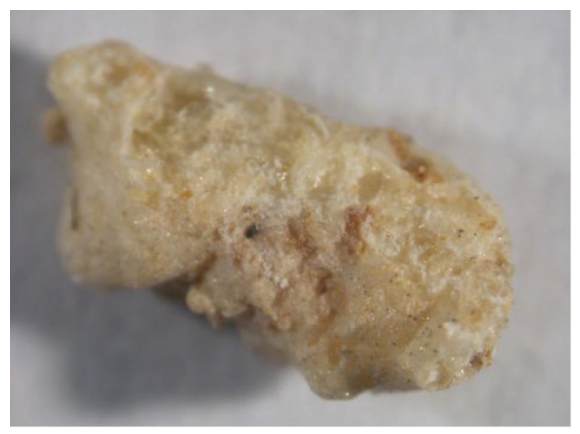

Figura 6: Micromuestra obtenida del lateral y fondo de la vasija MAM 3731 (García y Pérez 2011).

Figura 7: Análisis FTI de la vasija MAM 3731. Espectro de infrarrojos obtenido al analizar los fragmentos gruesos blanquecinos depositados en el interior de la botella MAM 3731 (García y Pérez 2011).

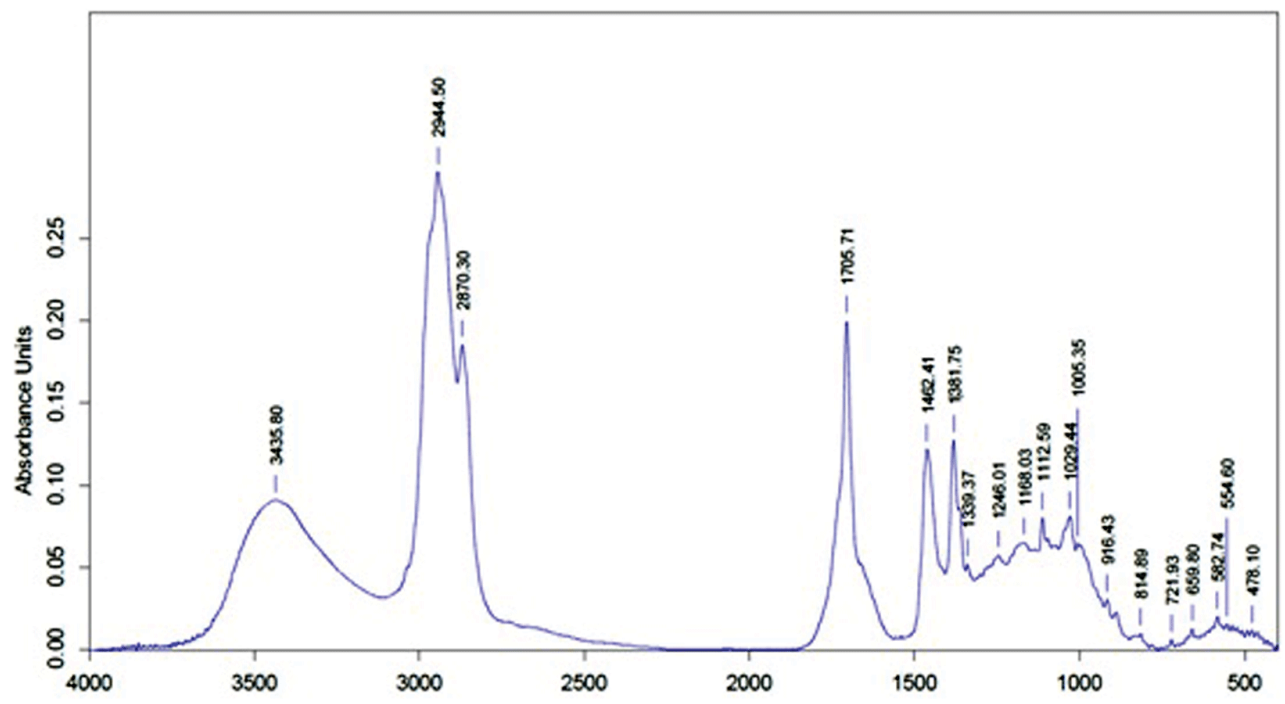




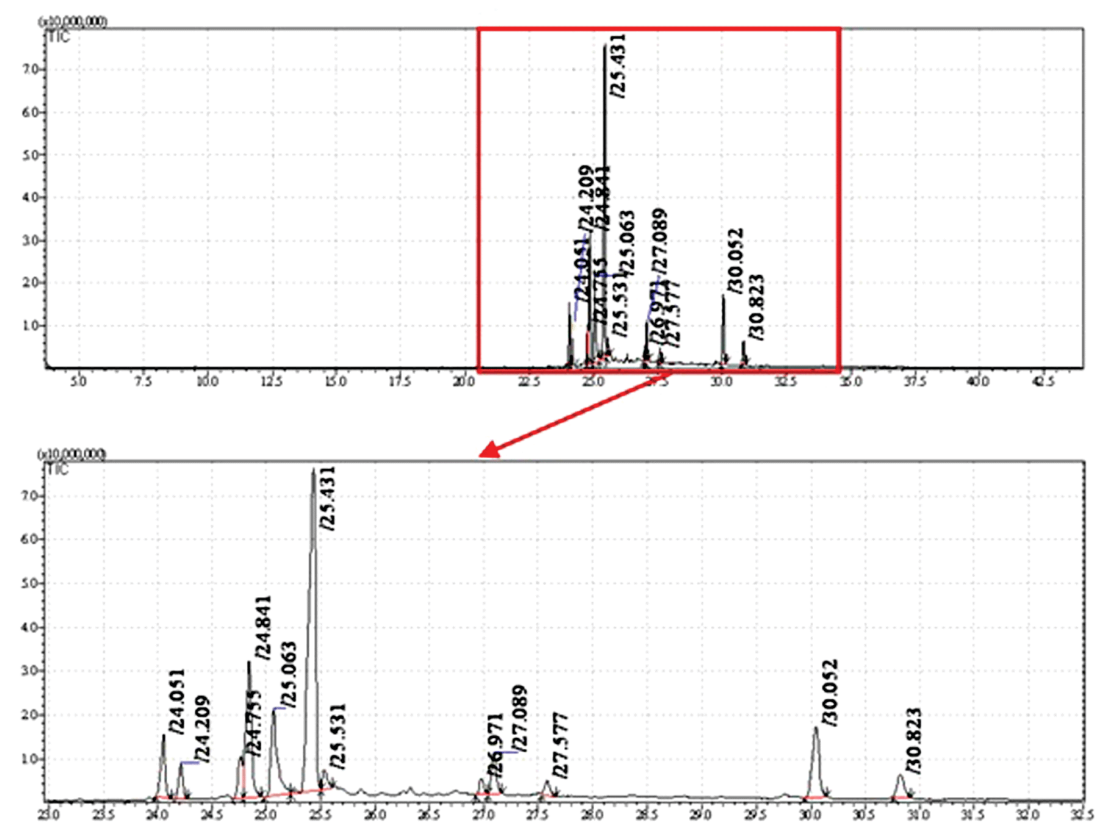

Figura 8: Porcentajes de los componentes caracterizados en diferentes momentos del análisis de la vasija MAM 3731.

pas planas y otras fibrosas, así como granos de polen. En todo caso, se concluye que, como suponíamos, efectivamente se trata de restos orgánicos vegetales.

Las bandas de absorción que se observan (Figura 7) indican la existencia de un componente terpénico, posiblemente triterpénico. Para confirmar el tipo de componente es necesario un segundo análisis mediante cromatografía de gases-espectrometría de masas.

\subsection{Cromatografia}

En el caso de la muestra Jama Coaque, la deposición y los principales picos cromatográficos se producen entre el minuto 24 y el 31 (Figura 8), que corresponden a compuestos triterpénicos ${ }^{14}$ probablemente procedentes de una planta, aunque no se puede confirmar a qué clase pertenece. En la figura $9 \mathrm{~b}$ se detalla ese rango señalado en la anterior.

Con más de $25 \%$ en la secuencia de picos encontramos componentes caracterizados como 67, 69, 79, 81, 91, 93, 95, 105, 107, 109, 203, 218, aunque Los picos más altos corresponden a $69,108,112,203,218,219$. El número 69 corresponde a un hidrocarburo insaturado, como los ácidos grasos insaturados. Los triterpenos son ácidos grasos, lípidos insaponificables ${ }^{15}$.

14 Triterpénico son terpenos de 30 carbonos, entre ellas saponinas, ceras vegetales o ácidos oléicos.

15 Lípidos insaponificables son algunos terpenos lípidos que se pueden transformar en jabones. 


\section{La muestra del interior}

Los restos adheridos al interior de la vasija MAM 3731 han revelado todo un universo microscópico que permite ir conociendo algo más de la cultura Jama Coaque. En su interior (Figura 9) se encuentran estructuras asombrosas, pero también se recogen almidones, fitolitos y pólenes que permiten la identificación de especies que han debido estar presentes en el proceso de fabricación de su contenido o forman parte del mismo.

De los cuatro tipos de moléculas orgánicas -glúcidos (almidón), lípidos (grasas), proteínas y ácidos nucleicos (ADN)-, se analizaron los dos primeros. Ante el interés despertado por el contenido de esta vasija, se decidió enviar otros fragmentos de la muestra a los laboratorios de la Universidad de Antioquia, Colombia, con la finalidad de identificar la presencia de almidones, y al Instituto del Patrimonio Cultural del Ecuador, donde se realizó también el estudio de pólenes.

\subsection{Glúcidos (almidones)}

El almidón de las plantas es un polisacárido de reserva alimenticia, formado por polímeros de glucosa (amilosa y amilopectina) que componen los hidratos de carbono
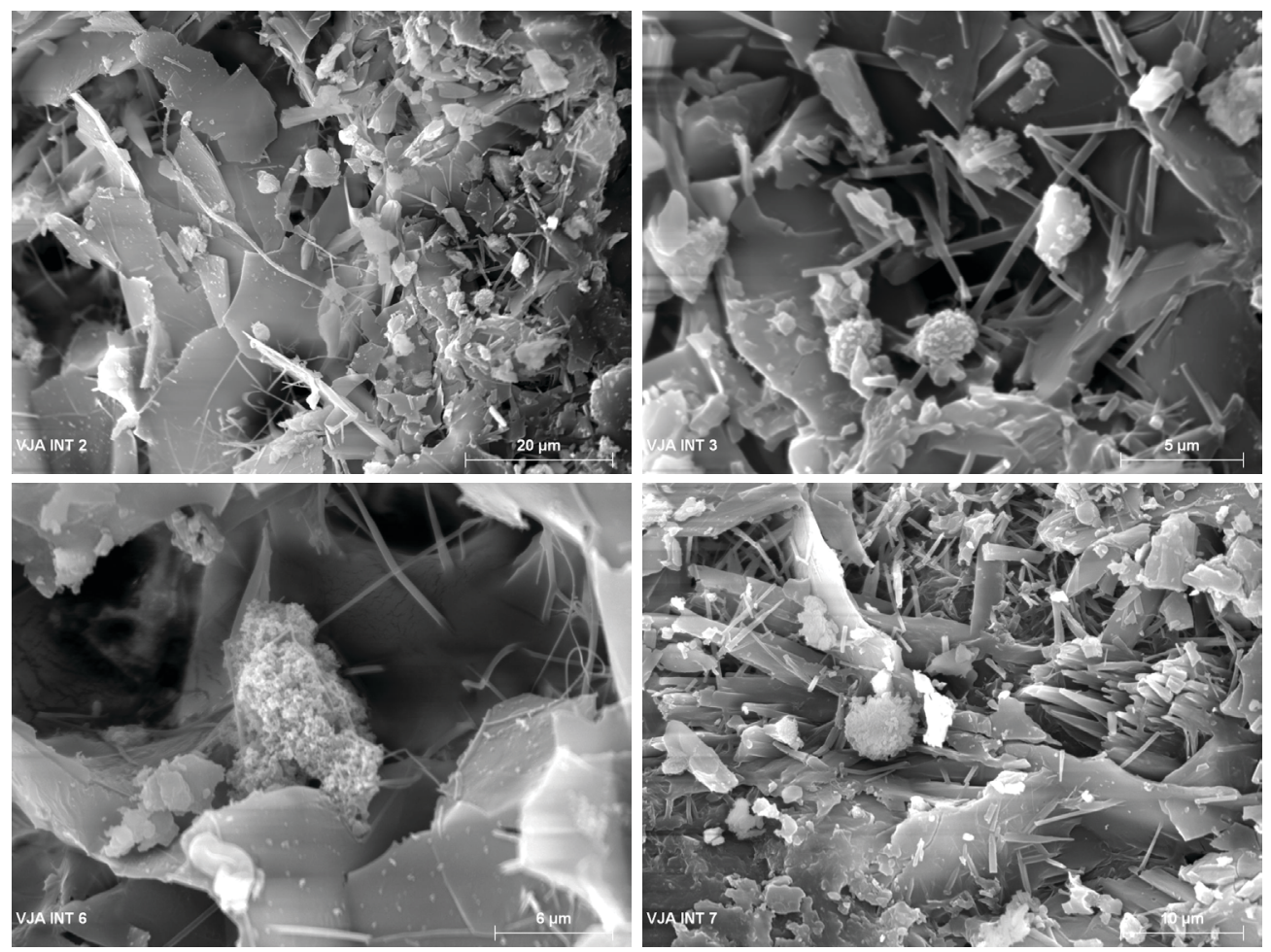

Figura 9: Vistas al microscopio electrónico del interior de la vasija MAM 3731 (García y Pérez 2011). 
Cuadro 1: Características de los ocho morfotipos que se identificaron de los 24 granos de almidón recuperados en la muestra de la vasija MAM 3731 (Aceituno y Lalinde 2011: 6).

\begin{tabular}{|c|c|c|c|c|c|c|c|c|}
\hline Morfotipo & 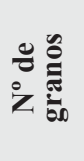 & 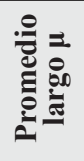 & 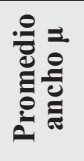 & = & 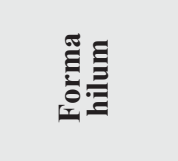 & 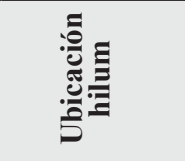 & 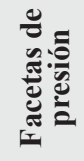 & 苞 \\
\hline Alveolar & 2 & 12,3 & 7,5 & $*$ & $*$ & $*$ & 0 & * \\
\hline Campaniforme & 4 & 7,3 & 6,8 & * & $\begin{array}{l}\text { U:1 } \\
\text { Punto: } 2\end{array}$ & Central: 3 & $\begin{array}{l}0,044 \\
0,084\end{array}$ & $*$ \\
\hline Circular & 6 & $*$ & $*$ & 9.6 & Punto: 4 & Central: 4 & 0,042 & 1 \\
\hline Irregular & 1 & 22,1 & 20,4 & $*$ & Estrellado: 1 & Central: 1 & 0,042 & * \\
\hline Ovalado & 3 & 14 & 13 & $*$ & Estrellado: 1 & Central: 1 & 0 & $*$ \\
\hline Ovoide & 2 & 8,5 & 6,1 & $*$ & Punto: 2 & $\begin{array}{l}\text { Central: } 1 \\
\text { Excéntrico: } 1\end{array}$ & 0 & 1 \\
\hline Poliédrico & 5 & 13,1 & 11,1 & $*$ & $\begin{array}{l}\text { Y: } 1 \\
\text { Estrellado: } 1 \\
\text { Circular: } 1 \\
\text { Punto: } 1\end{array}$ & Central: 4 & $\begin{array}{l}0,251 \\
0,210 \\
0,167\end{array}$ & $*$ \\
\hline Riñón & 1 & 12,5 & 10,8 & $*$ & $*$ & * & * & * \\
\hline
\end{tabular}

y pueden ser de dos tipos, los de asimilación, que ayudan a sintetizar el CO2 y los de reserva, que almacenan energía para las plantas (bulbos, tubérculos, semillas...).

El análisis de almidones de la vasija MAM 3731 fue encargado al laboratorio de Arqueología de la Universidad de Antioquía, Colombia, y llevado a cabo por Francisco Javier Aceituno y su equipo. Para la separación de los granos de almidón, se centrifugó la muestra a 2000 r.p.m. durante 15 minutos, disolviendo el residuo concentrado en agua pesada ${ }^{16}$ para separar dichos granos por flotación y de nuevo se centrifugó otros 5 minutos para separar las partículas más pesadas. Finalmente, se eliminó el agua pesada con agua destilada y nuevos centrifugados. El residuo se montó sobre placas para su observación al microscopio de 100 aumentos.

La identificación de almidones depende de las variables métricas (ancho, largo, diámetro) y morfológicas (forma, forma del hilum ${ }^{17}$, fisuras, facetas, bandas de crecimiento o cavidades centrales), que se comparan con tablas de referencia. Este suele ser el principal problema al tratar con culturas arqueológicas en contextos tropicales: la ausencia de tablas comparativas que permitan identificar especies.

Tras separar los granos de almidón y dimensionarlos, se compararon con las muestras de la colección de referencia, determinando la existencia de ocho morfotipos diferentes, entre los 24 granos de almidón que se recuperaron, cuyas características se definen en el informe (Cuadro 1). De estos ocho morfotipos que seguramente corresponderían a diferentes especies vegetales, se han podido reconocer tres taxones, sumando un total de siete gránulos, es decir, aproximadamente un 30\% del total recu-

16 El informe realizado por Francisco Javier Aceituno y Verónica Lalinde (2011), del Laboratorio de Arqueología de la Universidad de Antioquía describe con mayor detalle la metodología de trabajo aplicado al análisis de almidones.

17 El hilum o cruz de Malta, es el punto de nucleización a partir del que se forman los anillos o capas de almidón (Aceituno y Lalinde 2011). 

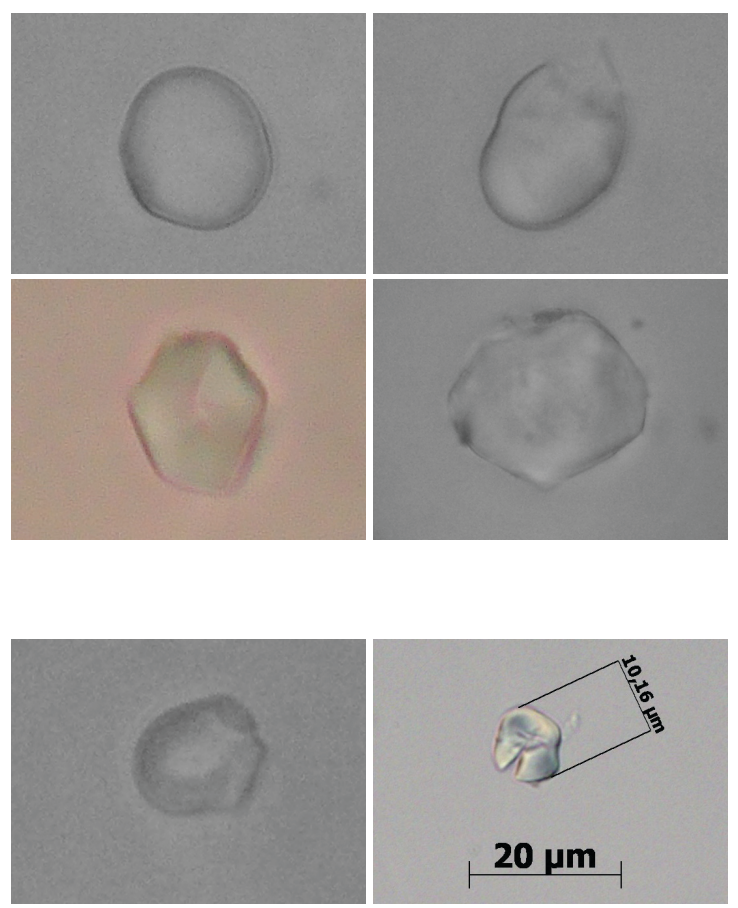

Figura 10: Granos de almidón identificados en la vasija MAM 3731; a: grano de almidón de cucurbitácea (Aceituno y Lalinde 2011: MU2 foto 29); b: grano de almidón de fabácea (ibid. : MU2 foto 49); c: grano de almidón de maíz (Zea mays) (ibid.: MU2 foto 8); d: grano de almidón de maíz (Zea mays) (ibid.: MU2 foto 23).

Figura 11: Almidón del género Bactris en la vasija MAM 3731 (Aceituno y Lalinde 2011: MU2 foto 54).

perado. Los taxones identificados son el maíz (Zea mays, 3 granos) y la cucurbitácea (3 granos), con un 12,5\% cada uno del total de la muestra, y un grano de almidón de arecaceae (Bactris gasipaes) que representa un 4,2\% (Figuras 10 y 11).

En relación con la identificación del grano de palma, se propone la especie Bactris cf gasipaes (familia Arecaceae) por la morfología del grano campaniforme, «con dos facetas de presión asimétricas en la corona de la campana, una ligera depresión central y un tamaño reducido de $5 \mu \mathrm{m}$ de largo» (Aceituno y Lalinde 2011: 6-7) (Figura 12). Los granos campaniformes con estas características son comunes en la especie B. gasipaes como se puede observar en la Figura 12a. La presencia de fitolitos y macrorestos de la familia Aracaceae en el registro arqueológico de la zona ocupada por la cultura Jama Coaque (Pearsall 1994a, 1994b, 1996, 2004) demuestra la convivencia y uso de esta especie de palma y refuerza su identificación en los residuos extraídos del recipiente cerámico.

Por otro lado, hay que destacar que los almidones recuperados de maíz no muestran alteraciones típicas del proceso de fermentación. Por tanto, el tipo de bebida que contenía esta botella no era una chicha, lo que ya suponíamos por su capacidad como contenedor.

\subsection{Fitolitos}

De esta misma muestra, otro fragmento fue enviado al Laboratorio del Instituto Nacional de Patrimonio Cultural del Ecuador (Guachamín 2011), donde identificaron 
Figura 12: Fitolitos identificados en la vasija MAM 3731; a: fitolito de Arecaceae, género Bactris; b: almidón de Bactris.

(Guachamín 2011).
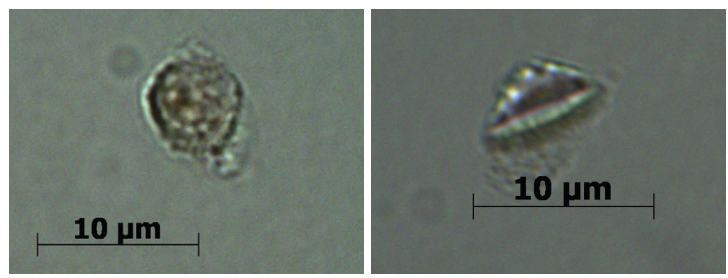

Figura 13: Polen en la vasija MAM 3731; a: polen deshidratado, diada, tamaño grande (Guachamín 2011); b: polen sin identificar (fotografiado por el Instituto de Patrimonio Cultural de España).

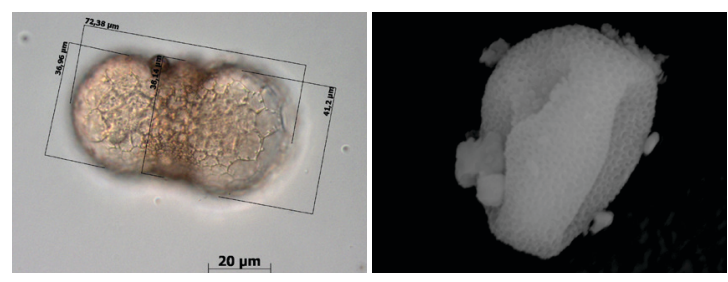

la presencia de fitolitos de palmera-Arecaceae, del género Bactris sp. (Figura 12a)que fueron además confirmados por el hallazgo de almidones de esta misma especie (Figura 12b).

\subsection{Pólenes}

Queda aún por conocer el contenido de los análisis polínicos (Figura 13). Una primera revisión identifica granos de pólenes procedentes de asteráceas, plantas de flores también identificadas en el estudio arqueobotánico del valle del Jama, pero demasiado comunes y genéricas para poder inferir algún dato. Es posible que alguno de los pólenes pertenezca al género Bactris $s p$.

\section{Interpretación}

El último paso consiste en la interpretación de los datos obtenidos del análisis de continente y contenido. Estas pequeñas botellas Jama Coaque muestran un elemento recurrente, que consiste en una estructura vegetal trifoliada. Iconográficamente tan solo ha sido posible precisar que se trata de una leguminosa con tubérculo (quizá ahipa). La propia botella fitomorfa pretende ser en realidad un tubérculo (Ver Gutiérrez Usillos 2011: fig. 88a y 88b). En su parte superior sobresalen cuatro ramas con el extremo trifoliado y botones perforados, es decir, se corresponde con la que hemos identificado como planta sagrada para la cultura Jama Coaque (Gutiérrez Usillos 2011: 177 y ss.).

A la vista de las evidencias, tanto de los taxones vegetales identificados a partir de almidones, fitolitos y pólenes, como de los resultados obtenidos por cromatografía ¿qué podemos decir del tipo de contenido portaban estas botellas y de su utilización? 
Hasta el momento, tan solo conocemos que se trata de un compuesto químico triterpénico característico de los aceites esenciales. Así que, un potente aceite diluido en una preparación compuesta por las principales plantas de cultivo (maíz, calabaza) y por la palma de chontaduro, y al menos otros cuatro tipos de vegetales, ninguno de los cuales corresponde a los cultivos más comunes reseñados en la región de Jama Coaque (yuca, frijol, maranta, o maní), posiblemente fuera consumida o más bien aplicada de forma individual para algún tipo de ritual.

También sabemos que no se trata de una bebida fermentada, ni una bebida alucinógena ya que no se recuperan trazas de substancias psicodélicas. El informe de Aceituno y Lalinde (2011: 11) señala que «los almidones recuperados de maíz no muestran alteraciones típicas del proceso de fermentación» descartándose, por tanto, que se trate de la chicha de maíz y chontaduro, lo que además tendría poco sentido en relación con la forma y tamaño de la botella.

Una posible interpretación es que contuviera algún tipo de brebaje purgante, pues el vómito es uno de los pasos esenciales en el complejo ceremonial de la toma de alucinógenos (Gutiérrez Usillos 2011: 213 y ss.) Pero, por otra parte, la identificación de componentes triterpénicos característicos de aceites esenciales sugiere más bien que estas botellitas se utilizaban para guardar substancias aceitosas. Estos aceites serían necesarios para poder aplicar los pigmentos minerales y vegetales que se usaban frecuentemente para decorar tanto el rostro como el cuerpo durante los rituales, en hombres y mujeres. En el Viejo Mundo son frecuentes los ungüentarios de diferentes formas y tamaños.

¿Podría tratarse de un contenedor de un aceite vegetal? Las semillas, por ejemplo los granos de maíz, tienen una proporción de humedad muy diferente al resto de la planta, que no llega al $20 \%$, predominando los carbohidratos, almidones y lípidos, generalmente triglicéridos. Entre un 3 y un 5\% del maíz es grasa vegetal, de tal forma que, como es sabido, uno de los aceites de cocina más utilizados hoy en día procede de este cereal. Mucho más productivo en este sentido es el fruto de la palmera Bactris sp., el chontaduro, cuyo mesocarpo o carne del fruto es muy rico en aceites. Su extracción es sencilla: simplemente se cuece el fruto en agua y por diferencia de densidad, al enfriar, se recoge en la superficie el aceite que es utilizado para alimento y quizá para estos otros menesteres. Los ácidos grasos obtenidos del mesocarpio del chontaduro incluyen aceites saturados (como palmítico y esteárico) y otros no saturados como palmitoléico, oléico, linoléico, o linoénico). Los de mayor proporción son el ácido oléico, que es casi el 50\% del total, y el palmítico, entre un 20 y un $40 \%$ (Orduz y Rangel 2002). Y como hipótesis para continuar investigando suponemos que si se utilizaba alguna variedad de ahipa (por el tipo de contenedor) que contuviera rotenona, este aceite podría actuar también como repelente de insectos, lo que evidentemente aportaría una función práctica a la estética.

Por tanto, pensamos que esta tipología de botellitas con tapón contenía el aceite realizado con estos productos vegetales, que permitía elaborar un tinte corporal, combinándolo sobre la palma de la mano u otro recipiente con ciertos pigmentos minerales. El aceite posibilitaría la fijación del pigmento a la piel, facilitaría su aplicación y extensión por el rostro o cuerpo de forma sencilla, en momentos ceremoniales. Al ser incoloro, este aceite podría utilizarse con diferentes pigmentos, ya que se ha constata- 
do a través de análisis iconográfico que en esta cultura se pintaban el rostro y cuerpo de diferentes colores, amarillo, rojo, negro y azul o azul verdoso.

En esas ceremonias se utilizarían también botellas silbato como las que se identificaron al inicio de este artículo, de manera que a partir de estos análisis arqueométricos, se han podido definir algunos pasos rituales en el complejo ceremonial Jama Coaque en los que se utilizaban dos tipologías de objetos cerámicos diferentes.

\section{Referencias bibliográficas}

Aceituno, Francisco Javier y Verónica LaLinde

2011 Informe. Análisis de almidones de la botella Jama Coaque MAM 3731. Laboratorio de Arqueología, Departamento de Antropología, Universidad de Antioquía. Manuscrito sin publicar.

Carvajal Barriga, E. Javier y Patricia Portero

2012 Arqueología microbiana: resucitando los testigos microscópicos de la historia de Quito. Editorial Académica Española.

García, María Antonia y Pedro Pablo Pérez

2011 Informe sobre Vasija Jama Coaque MAM 3731. Núm. Registro IPCE: 30660.7 de junio de 2011. Área de Laboratorio. Sección de Análisis de Materiales. Instituto de Patrimonio Cultural de España. Manuscrito sin publicar.

Guachamín, Ana

2011 Análisis de fitolitos y pólenes de la muestra de la botella Jama Coaque MAM 3731. Instituto Nacional de Patrimonio Cultural, Ecuador. Manuscrito sin publicar.

Gudemos, Mónica

2009 «El cuerpo del sonido: flautas antropomorfas de tradición Bahía». Revista Española de Antropología Americana 39 (1): 195-218.

GuTiÉRRez Usillos, Andrés

2010 Museología y documentación. Criterios para la definición de un proyecto de documentación en museos. Gijón: Trea.

2011 El eje del universo. Chamanes, sacerdotes y religiosidad en la cultura Jama Coaque del Ecuador prehispánico. Madrid: Ministerio de Cultura.

ORduz R., Javier Orlando y Jorge Alberto Rangel M.

2002 Frutas tropicales potenciales para el piedemonte llanero. Manual de Asistencia Técnica, 8. Villavicencio: Corporación Colombiana de Investigación Agropecuaria. http://www.corpoica.org.co/SitioWeb/Archivos/Publicaciones/Frutalestropicales cartilla.pdf, con acceso el 31/7/2012.

Pearsall, Deborah M.

1994a «Análisis macrobotánico», en Arqueología regional del norte de Manabí, Ecuador, Vol. 1, James A. Zeidler y Deborah M. Pearsall, eds., pp. 150-160. Pittsburg y Quito: University of Pittsburg y Ediciones Libri Mundi.

1994b «Análisis de fitolitos», en Arqueología regional del norte de Manabí, Ecuador, Vol. 1. James A. Zeidler y Deborah M. Pearsall, eds., pp. 162-174. Pittsburg y Quito: University of Pittsburg y Ediciones Libri Mundi. 
1996 «Reconstructing Subsistence in the Lowland Tropics. A Case Study from the Jama River Valley, Manabí, Ecuador», en Case Studies in Environmental Archaeology, Elizabeth J. Reitz, Lee A. Newsom y Sylvia J. Scudder, eds., pp. 233-254. Nueva York: Plenum Press.

2004 Plants and People in Ancient Ecuador: The Ethnobotany of the Jama River Valley. Belmont: Wadsworth.

PÉREZ DE ARCE, José

2004 «Análisis de las cualidades sonoras de las botellas silbadoras prehispánicas de los Andes». Boletín del Museo Chileno de Arte Precolombino 9: 9-34.

Zevallos, Carlos

1995 Nuestras raíces huancavilcas. Guayaquil: Casa de la Cultura Ecuatoriana Benjamín Carrión. 